\title{
Modificación de patrones cinemáticos y electromiográficos en extremidad inferior por el uso de celular \\ Modification of kinematic and electromyographic patterns in the lower limb by the use of cell phones \\ *Oscar Valencia, *María José Hudson, **Felipe Carpes, **Marcos Kunzler, *Fernanda Gándara, *Isidora Le Roy, *Rodrigo Guzmán-Venegas \\ *Universidad de los Andes (Chile), ** Universidade Federal do Pampa (Brasil)
}

Resumen. Las lesiones de transeúntes relacionadas al uso de teléfono celular han aumentado en relación con el total de accidentes peatonales. El objetivo de este estudio fue comparar variables cinemáticas y electromiográficas de ambas extremidades inferiores al enfrentar un obstáculo, con (CC) y $\sin$ (SC) el uso de celular. Diez mujeres jóvenes fueron evaluadas, las cuales caminaron y enfrentaron un obstáculo CC y SC. Con un modelo biomecánico 3D se evaluó la cinemática de extremidad inferior (plano sagital de cadera, rodilla, tobillo, junto al «toe clearance»). Al mismo tiempo se registró la actividad electromiográfica (EMG) de los siguientes músculos: tibial anterior (TA), gastrocnemio medial (GM), recto anterior (RA) y bíceps femoral (BF). Se calculó la amplitud EMG promedio de cada músculo, y el porcentaje de coactivación muscular entre: TA-GM y RA-BF. Se analizó la estrategia de ambas piernas, considerando un primer (P1) y segundo paso (P2) al cruzar el obstáculo, comparando entre una marcha CC vs CS. Según los resultados, la marcha CC incrementa el toe clearance, flexión de cadera, y la amplitud del GM, observado tanto en P1 como P2 al cruzar el obstáculo. Adicionalmente, el P2 reveló un incremento en la flexión de rodilla y tobillo. Por otro lado, la amplitud del TA y coactivación muscular entre TA-GM también aumentó CC en el P2. En conclusión, las variables cinemáticas y electromiográficas en las extremidades inferiores se modifican al cruzar un obstáculo CC. Estos hallazgos podrían indicar una estrategia protectora durante la tarea dual evaluada, minimizando el riesgo de caída.

Palabras clave: Electromiografía, cinemática, tarea dual, marcha con obstáculo.

\begin{abstract}
Pedestrian injuries related to the use of cell phone have increased in relation to the total number of pedestrian accidents. The aim of this study was to compare kinematic and electromyographic variables in both lower limbs at facing an obstacle, with (WC) and without (WoC) the use of a cell phone. Ten young women were evaluated, while walking and facing an obstacle WC and WoC. A 3D biomechanical model was used to evaluate the lower limb kinematics (hip, knee, ankle in the sagittal plane, together with «toe clearance»). At the same time, the electromyographic (EMG) activity was registered in the following muscles: tibialis anterior (TA), gastrocnemius medialis (GM), rectus femoris (RF) and biceps femoris (BF). The mean EMG amplitude of each muscle and the muscular coactivation percentage between: TA-GM and RA-BF were calculated. The strategy for both lower limbs considering the first (P1) and the second step (P2) were analyzed when crossing the obstacle, comparing between gait WC vs WoC. According to results, the gait WC increase the toe clearance, hip flexion, and the GM amplitude, observed both in P1 as P2 when the person crossed the obstacle. Furthermore, the P2 revealed an increase in the knee and ankle flexion. On the other hand, the TA amplitude and the muscular coactivation between TA-GM also increased WC in the P2. In conclusion, the kinematic and electromyographic variables in the lower limbs are modified when crossing an obstacle WC. These findings could indicate a protective strategy during the dual-task evaluated, minimizing the risk of falling. Key words: Electromyography, kinematics, dual task, gait with obstacle.
\end{abstract}

\section{Introducción}

Se ha descrito que el uso de teléfono celular durante la marcha es responsable de aumentar las exigencias cognitivas, generando un comportamiento inseguro y reduciendo la concentración frente a tareas duales debido a una falta de información visual (Lamberg \& Muratori, 2012). Esto incrementa el riesgo de tropezar, colisionar o generar lesiones a otros peatones (Licence, Smith, McGuigan, \& Earnest, 2015). Sumado a lo anterior, un estudio declara que $44 \%$ de las personas usa un teléfono celular mientras camina, provocando una reducción de la velocidad al cruzar la calle y transitar de forma involuntaria por lugares no señalizados (Hatfield \& Murphy, 2007; Nasar \& Troyer, 2013). Así mismo, una de las distracciones habituales por los peatones más jóvenes es responder un mensaje de texto o hablar por celular mientras caminan, según un estudio desarrollado en dos campus universitarios (observando un total de 10543 personas), comportamiento más frecuente en mujeres (Wells,

Fecha recepción: 20-02-20. Fecha de aceptación: 10-07-20

Oscar David Valencia Cayupán

ovalencia@uandes.cl
McClure, Porter, \& Schwebel, 2018).

Considerando la marcha como una tarea automática y adquirida durante la evolución humana (O’Neill et al., 2015), la demanda cognitiva requerida para enfrentar un obstáculo y al mismo tiempo desarrollar otra tarea, es muy alta. En consecuencia, si una persona posee una patología que altera el sistema somatosensorial, como la diabetes (Hsu, Liu, \& Lu, 2016), los accidentes peatonales pueden ser muy graves (Nasar \& Troyer, 2013). Así mismo, otro factor que influye directamente en el riesgo de sufrir un accidente mientras una persona camina, es el tiempo invertido en otra tarea de forma simultánea, por ejemplo, escribir un mensaje de texto en un celular (Shigeru Haga et al., 2015). A lo anterior, se suma la dificultad de enfrentar diferentes obstáculos que se pueden presentan en la vía pública, tales como: bordillos, escalones, rampas, entre otros (Licence et al., 2015).

Según la literatura, algunos estudios han descrito cambios en variables biomecánicas vinculadas a la marcha y el desarrollo simultáneo de otra tarea al enfrentar un obstáculo, determinando patrones protectores que minimizan el riesgo de una caída (Al-Yahya et al., 2011; Licence et al., 2015). Esto considera la modificación de algunos parámetros temporoespaciales como la velocidad, longitud del paso, frecuencia 
del paso (Licence et al., 2015; Al-Yahya et al., 2011) y el «toe clearance» (descrito como la distancia vertical mínima entre un punto de la región anterior del pie y la superficie del suelo, durante la fase de vuelo) (Dadashi et al., 2013). En consecuencia, las variables que caracterizan la marcha humana han sido consideradas como complejas, debido al constante ajuste sensorial y cognitivo (Al-Yahya et al., 2011).

Un estudio desarrollado por MacLellan (2017), el cual compara las variaciones cinemáticas y electromiográficas de las extremidades inferiores con y sin la incorporación de un obstáculo, señala la presencia de un ajuste locomotor de ambas extremidades. Sus hallazgos reportan mayores ángulos articulares de cadera, rodilla y tobillo al pasar un obstáculo. Adicionalmente, describe características asimétricas al comparar la primera extremidad inferior que enfrenta el obstáculo versus la extremidad contrataleral. Esto también ocurre con los patrones de activación muscular de veintiocho músculos evaluados (MacLellan, 2017). Sin embargo, existe poca evidencia del efecto generado por el uso del celular al enfrentar un obstáculo, y la modificación de parámetros biomecánicos durante la locomoción humana. El propósito de esta investigación fue comparar variables cinemáticas y electromiográficas de ambas extremidades inferiores al enfrentar un obstáculo, con y sin el uso de celular. Nuestra hipótesis de trabajo planteó que el uso de celular genera una mayor amplitud de la actividad electromiográfica, y un incremento de los grados de movimiento en extremidades inferiores, al cruzar un obstáculo

\section{Material y métodos}

Mediante un estudio analítico de corte transversal, se incluyeron solo mujeres jóvenes entre 25 y 34 años. Todas las participantes firmaron previamente un consentimiento informado, aprobado por un Comité de Ética local (folio: CEC201602). El tamaño muestral mínimo fue calculado considerando los valores promedios de «toe clearance» obtenidos por License et al. (2015) durante la marcha con y sin el uso de celular (111 $\pm 19 \mathrm{~mm}$ y $94 \pm 13 \mathrm{~mm}$, respectivamente). Para esto se utilizó una potencia de $80 \%$ y alfa de 0.05 , arrojando una muestra mínima de 8 personas. Este cálculo fue desarrollado en el software G-Power (versioìn 3.1.9.2, Universitat Kiel,Alemania).

Todas las voluntarias utilizaron su propio celular (con pantalla táctil), considerando un tiempo mínimo de pertenencia igual o mayor a 3 meses. Por otro lado, se excluyeron aquellas mujeres con lesión de extremidad inferior en los últimos 6 meses, con patologías neurológicas, problemas visuales no corregidos y cualquier otra alteración motora que impida el desarrollo de una marcha normal.

\section{Procedimiento}

Las participantes caminaron una distancia de $8 \mathrm{~m}$ a una velocidad autoseleccionada (Guadagnin, Rocha, Bobbert, Duysens, \& Carpes, 2020; Valencia, Araneda, Cárcamo, Carpes, \& Guzmán-Venegas, 2018). A los 4 metros se instaló un obstáculo (dimensiones: $15 \mathrm{~cm}$ de ancho, $60 \mathrm{~cm}$ de largo), el cual se ajustó previamente al $10 \%$ de la longitud de la extremidad inferior de cada voluntaria (determinada por la distancia vertical desde el trocánter mayor hasta el suelo).
Este porcentaje fue considerado producto de las asimetrías articulares reportadas por otras investigaciones (Hsu et al., 2016; Pan, Hsu, Chang, Renn, \& Wu, 2016). Los datos cinemáticos y electromiográficos fueron sincronizados con el software de captura y análisis Nexus (versión 2.6, Vicon ${ }^{\circledR}$, UK). Para estimar las variables cinemáticas se utilizaron 16 marcadores reflectantes ubicados en puntos anatómicos según el modelo Plug-in Gait (Kadaba et al 1990), los cuales fueron registrados con un sistema de análisis del movimiento 3D compuesto por 8 cámaras infrarrojas (T-Series, Vicon Motion Systems, Oxford, UK). La captura fue ajustada a una frecuencia de $200 \mathrm{~Hz}$. De forma adicional, se agregaron dos marcadores reflectantes ubicados en el punto más anteroinferior del hallux (definidos como Pa), para ambos pies (figura 1), y dos marcadores en la región superior del obstáculo (definidos como Mo). Estos se utilizaron para determinar el «toe clearance»y otros parámetros.

La actividad electromiográfica (EMG) fue registrada con un sistema Bagnoli de 16 canales (Delsys, Inc., Boston, USA), utilizando una frecuencia de muestreo de $1000 \mathrm{~Hz}$ y un filtro analógico pasa banda entre $20-500 \mathrm{~Hz}$. Los electrodos de superficie (bipolar $\mathrm{Ag} / \mathrm{AgCl}$, dimensión de contacto $=10 \mathrm{x} 1 \mathrm{~mm}$, distancia inter-electrodo de $10 \mathrm{~mm}$ ) fueron ubicados de forma bilateral según la recomendación SENIAM (Hermens et al., 1999), en los siguientes músculos: recto anterior (RA), bíceps femoral (BF), gastrocnemio medial (GM) y tibial anterior (TA). Previamente, las regiones delimitadas en la piel fueron rasuradas y límpiadas con alcohol (desnaturalizado al 95\%) y algodón. Adicionalmente, se registraron tres contracciones isométricas voluntarias máximas (CVM) por cada músculo (considerando 5 segundos de contracción y $1 \mathrm{mi}$ nuto de descanso). Esto se desarrolló para ajustar las señales electromiográficas a la CVM y expresarlas en porcentajes.

Cada voluntaria caminó con y sin el uso de un celular (CC y SC, respectivamente). El uso del celular consistió en responder una serie de preguntas de baja complejidad, enviadas segundos antes de iniciar la marcha (vía WhatsApp). Esto exigía de forma simultánea la creación de un mensaje de texto y sobrepasar el obstáculo. El orden de ejecución de la tarea fue asignado de manera aleatoria. Se consideraron 3 pruebas válidas para ambas condiciones (esto fue confirmado por un investigador, quien aseguraba el uso del celular, y la recepción de la respuesta de cada voluntaria), las cuales posteriormente fueron procesadas y representadas por el promedio de cada variable.

\section{Procesamiento de datos}

El procesamiento de las variables electromiográficas (RF, BF, GM y TA) fue estimada a partir de una ventana definida por el contacto inicial (Ci) del pie hasta el momento en que existe una coincidencia vertical entre las trayectorias de los marcadores Pa y Mo (figura 1). Este procedimiento fue considerado para ambas extremidades inferiores, respetando el pie seleccionado (derecho o izquierdo) por el voluntario para cruzar de forma inicial el obstáculo. Así se definió el primer y segundo paso. Cada señal electromiográfica fue rectificada y filtrada con un pasa bajo de $20 \mathrm{~Hz}$ (tipo Butterworth), cuarto orden (Flores-Leon, Soto, Araneda, Guzman-Venegas, \& De La Rosa, 2019; Valencia et al., 2020). Luego, fueron ajusta- 


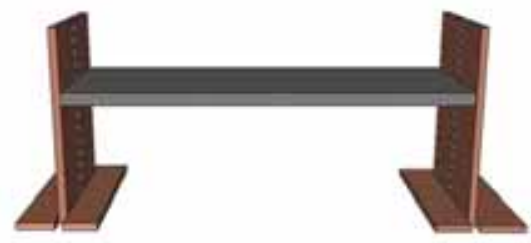

\section{B}

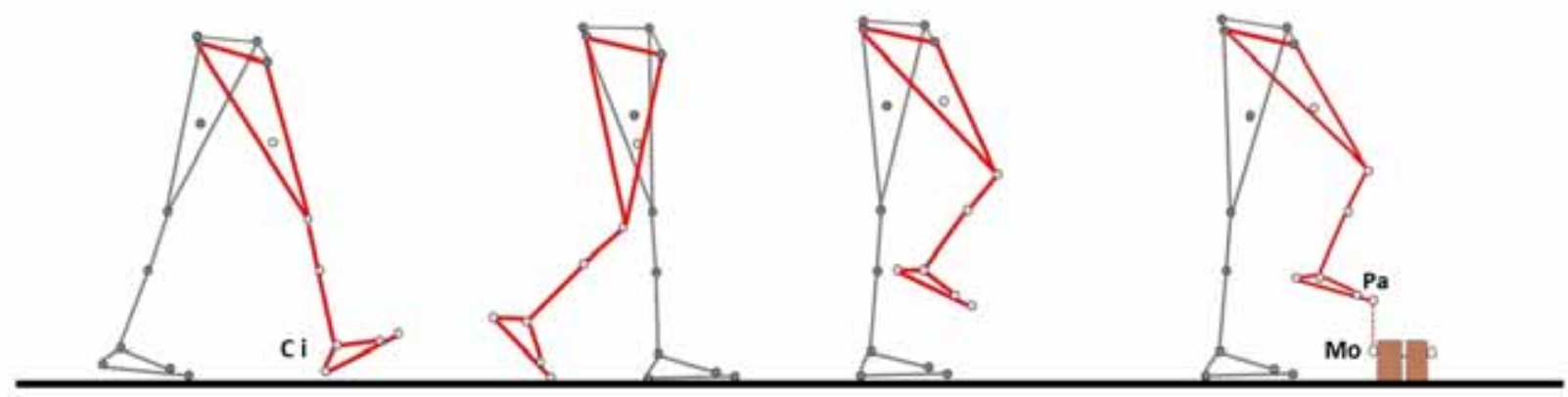

Figura 1: A. Vista frontal del obstáculo; B. Muestra la secuencia de la extremidad inferior derecha (color rojo) al momento de enfrentar el obstáculo, considerada como ventana de análisis para el procesamiento de la actividad electromiográfica (desde el contacto inicial (Ci) hasta el instante en que la trayectoria de los marcadores Pa y Mo se sincronizan de forma vertical).

das a la CVM y representadas por la amplitud promedio de la ventana analizada (expresada como \%CVM).

Para calcular el porcentaje de coactivación muscular se utilizó la fórmula de Falconer \& Winter (1985):

\%coactivación= $(($ área común $\mathrm{A} \& \mathrm{~B}) /($ área $\mathrm{A}+$ área B $))$ x 100\%

donde $\mathrm{A}$ y $\mathrm{B}$ representan a dos músculos antagonistas, considerando el cociente entre el área común de A\&B y la sumatoria de las áreas A y B, multiplicado por 100. Utilizando lo anterior, se calcularon las coactivaciones para los siguientes pares musculares: TA-GM y RA-BF. Estos también fueron expresados como porcentajes.

Por otro lado, las variables cinemáticas fueron extraídas a partir de la coincidencia vertical entre la trayectoria de los marcadores Pa y Mo, para cada extremidad inferior (figura 1). En esta oportunidad se consideraron los grados de movimiento articular en el plano sagital para cadera, rodilla y tobillo, como también el valor del toe clearance (distancia vertical entre los marcadores $\mathrm{Pa}$ y Mo, en ambas extremidades inferiores) expresado en milímetros. Los valores positivos representaron los grados de flexión de las articulaciones mencionadas. Considerando el mismo instante temporal, se registró la velocidad del marcador Pa (expresado en $\mathrm{m} / \mathrm{s}$, para ambos pies) utilizando como referencia el desplazamiento antero-posterior.

\section{Análisis estadístico}

El total de la muestra evaluada fue caracterizado utilizando el promedio y desviación estándar de datos demográficos. Se aplicó la prueba de Shapiro-Wilk para evaluar los supuestos de normalidad de las siguientes variables: toe clearance, cinemática articular (cadera, rodilla y tobillo), actividad electromiográfica de cada músculo (RA, BF, GM y TA), niveles de coactivación (TA-GM y RA-BF) y velocidad del marcador Pa. Posteriormente, se utilizó la prueba t-student o Wilcoxon para comparar las variables mencionadas, con y sin el uso de celular al enfrentar el obstáculo. Todos los análisis estadísticos fueron desarrollaron en el software GraphPad Prism (versión 8.3.1, USA), considerando un valor $\mathrm{p}<.05$, a una cola.

\section{Resultados}

Se evaluó un total de 10 mujeres jóvenes (edad $=27.9 \pm 3.81$ años, masa $=58.16 \pm 4.02 \mathrm{~kg}$, estatura $=1.62 \pm 0.05 \mathrm{~m}, \mathrm{IMC}: 21.9 \pm 1.8$ $\mathrm{kg} / \mathrm{m}^{2}$ ). Siete de las voluntarias utilizaron la extremidad inferior derecha como primer paso para enfrentar el obstáculo, mientras que el resto lo hizo con la extremidad inferior izquierda.

\section{Cinemática}

El primer paso que enfrentó el obstáculo mostró un aumento estadísticamente significativo del toe clearance con elusodel celular $(\mathrm{SC}=142.10 \pm 40.65 \mathrm{~mm}, \mathrm{CC}=194.60 \pm 47.84 \mathrm{~mm}$, $p=.0003$ ). Lo mismo se observó en el rango de flexión de cadera, el cual se incrementa con el uso del celular $\left(\mathrm{SC}=53.75 \pm 6.31^{\circ}, \mathrm{CC}=60.94 \pm 6.99^{\circ}, p=.0006\right)$. Por el contrario,

\begin{tabular}{|c|c|c|c|c|c|}
\hline \multirow{3}{*}{ Primer paso } & \multirow{2}{*}{\multicolumn{2}{|c|}{$\mathrm{SC}(\mathrm{n}=10)$}} & & & \\
\hline & & & \multicolumn{3}{|c|}{$\mathrm{CC}(\mathrm{n}=10)$} \\
\hline & & & & & \\
\hline Flexión / extensión de tobillo $\left(^{\circ}\right)$ & $10.44(4.62)$ & 10.80 & $11.27(4.21)$ & 9.82 & .1382 \\
\hline Flexión de rodilla $\left({ }^{\circ}\right)$ & $86.08(16.94)$ & 83.59 & $88.53(16.20)$ & 86.67 & .5042 \\
\hline Flexión de cadera $\left({ }^{\circ}\right)$ & $53.75(6.31)$ & 53.24 & $60.94(6.99)$ & 59.49 & $.0006 *$ \\
\hline Toe Clearance $(\mathrm{mm})$ & $142.10(40.65)$ & 142.90 & $194.60(47.84)$ & 188.90 & $.0003 *$ \\
\hline Velocidad de $\mathrm{Pa}(\mathrm{m} / \mathrm{s})$ & $3.66(0.31)$ & 3.57 & $3.50(0.50)$ & 3.45 & .1883 \\
\hline Segundo paso & & & & & \\
\hline Flexión / extensión de tobillo $\left({ }^{\circ}\right)$ & $-11.94(5.71)$ & -11.53 & $-5.137(8.67)$ & -4.50 & $.0048^{*}$ \\
\hline Flexión de rodilla $\left({ }^{\circ}\right)$ & $78.15(4.89)$ & 78.13 & $94.06(8.24)$ & 93.95 & $.0002 *$ \\
\hline Flexión de cadera $\left({ }^{\circ}\right)$ & $16.44(5.85)$ & 16.80 & $24.35(8.42)$ & 25.89 & $.0002 *$ \\
\hline Toe Clearance (mm) & $85.62(34.50)$ & 81.15 & $150.3(56.92)$ & 139.40 & $.0025 *$ \\
\hline Velocidad de $\mathrm{Pa}(\mathrm{m} / \mathrm{s})$ & $2.02(0.36)$ & 1.95 & $2.38(0.18)$ & 2.41 & $.0048^{*}$ \\
\hline
\end{tabular}


no se observaron diferencias significativas en la articulación de rodilla y tobillo ( $p=.5042 ; p=.1382$, respectivamente), asociadas al primer paso (Tabla 1 ).

En relación con el segundo paso, la longitud del toe clearance aumenta de forma significativa al utilizar celular $(\mathrm{SC}=85.62 \pm 34.50 \mathrm{~mm}, \mathrm{CC}=150.30 \pm 56.92 \mathrm{~mm}, p=.0025)$. De la misma forma, la cinemática de todas las articulaciones mostraron diferencias estadísticamente significativas al enfrenar el obstáculo con celular. Describiendo un aumento en la flexión de cadera $\left(\mathrm{SC}=16.44 \pm 5.85^{\circ}, \mathrm{CC}=24.35 \pm 8.42^{\circ}, p=.0006\right.$ ), rodilla $\left(\mathrm{SC}=78.15 \pm 4.89^{\circ}, \mathrm{CC}=90.06 \pm 8.24^{\circ}, p=.0002\right) \mathrm{y}$ disminuyendo la extensión de tobillo $\left(\mathrm{SC}=-11.94 \pm 5.71^{\circ}, \mathrm{CC}=\right.$ $5.13 \pm 8.67^{\circ}, p=.0048$; Tabla 1). Adicionalmente, la velocidad del Pa en relación con el primer paso que cruza el obstáculo no muestra diferencias estadísticas al comparar entre tareas $(\mathrm{SC}=3.66 \pm 0.31 \mathrm{~m} / \mathrm{s}, \mathrm{CC}=3.50 \pm 0.50 \mathrm{~m} / \mathrm{s}, p=.1883)$, sin embargo, al desarrollar la misma comparación considerando el segundo paso, este sí reporta diferencias significativas, indicando una mayor velocidad en la condición CC $(\mathrm{SC}=2.02 \pm 0.36 \mathrm{~m} / \mathrm{s}, \mathrm{CC}=2.38 \pm 0.18 \mathrm{~m} / \mathrm{s}, p=.0048)$.

\section{Actividad EMG promedio}

El valor promedio de la actividad electromiográfica tuvo un aumento significativo para el músculo GM con el uso de celular durante el primer paso $(\mathrm{SC}=26.98 \pm 13.54 \% \mathrm{CVM}$, $\mathrm{CC}=30.46 \pm 14.77 \% \mathrm{CVM}, p=.0137$ ) (Tabla 2). Algo diferente muestran los resultados en relación al segundo paso, donde sólo el músculo TA describe diferencias significativas ( $\mathrm{SC}=11.17 \pm 3.64 \% \mathrm{CVM}, \mathrm{CC}=13.37 \pm 4.76 \% \mathrm{CVM}, p=.0459$ ), el cual aumenta su actividad promedio con el uso de celular (Tabla 2).

\section{Coactivación muscular}

Tabla 2

Actividad electromiográfica promedio, normalizada a la contracción voluntaria máxima de cada músculo $(\mathrm{RA}=$ recto anterior, $\mathrm{BF}=$ biceps femoral, TA=tibial anterior y $\mathrm{GM}=$ gastrocnemio medial), evaluada en ambas extremidades inferiores. Representada en base al primer y segundo paso, al enfrentar un obstáculo con (CC) y $\sin$ (SC) el uso de celula

\begin{tabular}{|c|c|c|c|c|c|}
\hline & \multicolumn{2}{|c|}{$\mathrm{SC}(\mathrm{n}=10)$} & \multicolumn{3}{|c|}{$\mathrm{CC}(\mathrm{n}=10)$} \\
\hline & Promedio (DE) & Mediana & Promedio (DE) & Mediana & $p$-value \\
\hline \multicolumn{6}{|l|}{ Primer paso } \\
\hline RA (\%CVM) & $12.70(11.52)$ & 6.83 & $12.61(8.83)$ & 9.71 & 2158 \\
\hline BF (\%CVM) & $13.25(11.46)$ & 9.99 & $12.77(6.34)$ & 12.63 & . 1377 \\
\hline TA $(\% \mathrm{CVM})$ & $12.01(3.33)$ & 11.53 & $12.74(3.34)$ & 12.00 & .1444 \\
\hline GM (\%CVM) & $26.98(13.54)$ & 21.08 & $30.46(14.77)$ & 24.45 & $.0137 *$ \\
\hline \multicolumn{6}{|l|}{ Segundo paso } \\
\hline RA (\%CVM) & $12.76(12.84)$ & 7.31 & $11.61(10.48)$ & 74.05 & . 0967 \\
\hline $\mathrm{BF}(\% \mathrm{CVM})$ & $17.49(11.70)$ & 15.30 & $15.55(6.87)$ & 14.94 & .4609 \\
\hline TA (\%CVM) & $11.17(3.64)$ & 12.62 & $13.37(4.76)$ & 13.09 & $.0459 *$ \\
\hline GM (\%CVM) & $24.4(6.53)$ & 25.36 & $23.01(6.11)$ & 22.08 & .1082 \\
\hline
\end{tabular}

Los porcentajes de coactivación muscular muestran únicamente diferencias significativas en el par muscular TA$\mathrm{GM}(\mathrm{SC}=33.97 \pm 10.43 \%, \mathrm{CC}=39.53 \pm 10.27 \%, p=.0292)$, el cual aumentó con el uso de celular, sólo para el segundo paso que enfrentó el obstaìculo (Tabla 3).

\section{Tabla 3}

Porcentajes de coactivación muscular en extremidades inferiores (recto anterior- bíceps femoral: RA- BF, tibial anterior-gastrocnemio medial: TA-GM), representada para el primer y segundo paso al enfrentar un obstáculo con (CC) y $\sin$ (SC) el uso de celular. $\frac{\text { paso al enfrentar un obstáculo con (CC) y } \sin (\mathrm{SC}) \text { el uso de celular. }}{\mathrm{SC}(\mathrm{n}=10)}$

\begin{tabular}{lccccc}
\hline \multicolumn{5}{c}{$\mathrm{SC}(\mathrm{n}=10)$} & \multicolumn{2}{c}{$\mathrm{CC}(\mathrm{n}=10)$} \\
\hline & Promedio (DE) & Mediana & Promedio (DE) & Mediana & $p$-value \\
\hline Primer paso & & & & & \\
RA -BF (\%) & $50.23(15.99)$ & 49.86 & $53.80(14.47)$ & 58.55 & .1945 \\
TA -GM (\%) & $29.69(10.05)$ & 31.53 & $28.97(9.251)$ & 26.97 & .7359 \\
Segundo paso & & & & & \\
RA-BF (\%) & $36.71(6.93)$ & 35.93 & $39.80(8.47)$ & 38.85 & .2874 \\
TA-GM (\%) & $33.97(10.43)$ & 30.81 & $39.53(10.27)$ & 35.61 &. $\mathbf{0 2 9 2 *}$ \\
\hline *p< .05; DE: desviación estándar. & & & &
\end{tabular}

\section{Discusión}

El objetivo principal de este estudio fue comparar variables cinemáticas y electromiográficas (EMG) de ambas extremidades inferiores al enfrentar un obstáculo, con y sin el uso de celular. Como resultado se observó una variación en la estrategia de cada paso al enfrentar el obstáculo, comparando la condición $\mathrm{CC}$ vs SC, las cuales describen un patrón diferente al analizar las extremidades por separado.

En consecuencia, el análisis cinemático del primer paso mostró un aumento significativo del toe clearance durante el enfrentamiento del obstáculo CC, aparentemente explicado por el incremento de la flexión de cadera. Sin embargo, al analizar las señales EMG del músculo RA, no se observaron cambios significativos en base a su amplitud promedio, por consiguiente, el aumento de la flexión de cadera podría estar vinculado a la acción de otro músculo o grupo muscular, por ejemplo, el psoas iliaco. Por otro lado, se observó un aumento significativo en el promedio de actividad electromiográfica del músculo GM con el uso del celular, a pesar de no encontrar cambios en los grados de movimiento de la rodilla y el tobillo. Este aumento de la actividad EMG podría atribuirse a una preparación de la fase de push up en la marcha, cuyo objetivo sería acumular energía elástica para enfrentar el obstáculo (Gianakos, Yasui, Murawski, \& Kennedy, 2016).

En relación con la variación de la velocidad del marcador Pa ubicado a nivel del hallux, este solo reportó diferencias significativas en base al segundo paso. Esta variable describe un aumento en su magnitud CC, asociada tal vez con el incremento significativo del toe clearance de la misma extremidad. Esto podría reflejar un efecto adquirido del segundo paso y, en consecuencia, un mayor riesgo de tropezar con el obstáculo. Por otro lado, los altos valores de velocidad asociados al marcador Pa podrían estar vinculados a los cambios bruscos generados por la flexo-extensión del tobillo al momento de cruzar el obstáculo (MacLellan, 2017).

El segundo paso, reportó cambios significativos en los grados de movimiento de todas las articulaciones de extremidad inferior y el toe clearance CC. Lo anterior describió un patrón común en base a la segunda extremidad que cruza el obstáculo, observando un aumento de la flexión de rodilla, cadera y disminución de la flexión dorsal de tobillo. En consecuencia, esto también generó un incremento del toe clearance, lo cual podría reflejar un tipo de ajuste sensorial vinculado a una mayor demanda visual al escribir un mensaje de texto en el celular mientras se genera la locomoción, reportado previamente por la literatura (Chen, Lo, Kay, \& Chou, 2018). Adicionalmente, el segundo paso describió una mayor activación del músculo TA y coactivación del par muscular TA-GM, con el uso de celular. Esto podría atribuir un incremento en la estabilidad del tobillo, debido a la pérdida visual del segundo paso al cruzar el obstáculo. Algo similar fue reportado por Pijnappels et al. (2006), quienes describieron un aumento de la amplitud EMG del TA como respuesta anticipatoria al enfrentar un obstáculo.

Los hallazgos observados en este estudio podrían ser el resultado de estrategias motoras en mujeres jóvenes para generar adaptaciones durante el desarrollo de una tarea dual. Esto podría confirmar el desarrollo de un patrón protector durante la marcha humana (Al-Yahya et al., 2011; Licence et 
al., 2015), más importante que escribir un mensaje de texto, categorizada como una tarea dual de baja complejidad (Caramia, Bernabucci, D’Anna, De Marchis, \& Schmid, 2017). Lo anterior concuerda con la teoría basada en la competencia de los requerimientos de atención frente a tareas duales (Yogev-Seligmann et al., 2010), donde la prioridad aún está en discusión (Shumway-Cook, Woollacott, Kerns, \& Baldwin, 1997). Según lo anterior, el desafío de futuras investigaciones podría estar vinculado al desarrollo de estas evaluaciones en adultos mayores, debido al gran porcentaje de caídas reportadas en accidentes de tránsito y el deterioro progresivo de los sistemas sensoriales (Dadashi et al., 2013; Guadagnin, da Rocha, Duysens, \& Carpes, 2016).

Este estudio no está exento de limitaciones. Una de ellas corresponde al ambiente controlado donde se desarrollaron las pruebas, distinto al escenario habitual de un peatón que cruza la calle. Junto a esto, el enfrentamiento a un obstáculo ubicado en el mismo lugar y no de forma accidental, podría considerarse como una limitación del protocolo experimental. Adicionalmente, otras limitaciones que deben ser consideradas son: la complejidad de las preguntas y respuestas enviadas por mensaje de texto, las cuales no fueron medidas, y la cantidad de personas evaluadas.

\section{Conclusión}

Considerando la muestra evaluada, se observó un aumento en los grados de movimiento y niveles de amplitud electromiográfica al cruzar un obstáculo y responder un mensaje de texto en un celular. Las estrategias observadas para el primer y segundo paso difieren tanto en variables cinemáticas como electromiográficas. Sin embargo, el aumento del toe clearance $\mathrm{CC}$ se describe para ambas extremidades inferiores al cruzar el obstáculo.

\section{Referencias}

Al-Yahya, E., Dawes, H., Smith, L., Dennis, A., Howells, K., \& Cockburn, J. (2011). Cognitive motor interference while walking: A systematic review and meta-analysis. Neuroscience \& Biobehavioral Reviews, 35(3), 715-728. https://doi.org/10.1016/j.neubiorev.2010.08.008

Caramia, C., Bernabucci, I., D’Anna, C., De Marchis, C., \& Schmid, M. (2017). Gait parameters are differently affected by concurrent smartphone-based activities with scaled levels of cognitive effort. PLOS ONE, 12(10), e0185825. https://doi.org/10.1371/ journal.pone. 0185825

Chen, S.-H., Lo, O.-Y., Kay, T., \& Chou, L.-S. (2018). Concurrent phone texting alters crossing behavior and induces gait imbalance during obstacle crossing. Gait \& Posture, 62, 422-425. https://doi.org/ 10.1016/j.gaitpost.2018.04.004

Dadashi, F., Mariani, B., Rochat, S., Büla, C., Santos-Eggimann, B., \& Aminian, K. (2013). Gait and Foot Clearance Parameters Obtained Using Shoe-Worn Inertial Sensors in a Large-Population Sample of Older Adults. Sensors, 14(1), 443-457. https://doi.org/10.3390/ s140100443

Falconer, K., \& Winter, D. A. (1985). Quantitative assessment of cocontraction at the ankle joint in walking. Electromyography and Clinical Neurophysiology, 25(2-3), 135-149. Retrieved from http:/ /www.ncbi.nlm.nih.gov/pubmed/3987606

Flores-Leon, A. F., Soto, V., Araneda, O. F., Guzman-Venegas, R., \& De La Rosa, F. J. B. (2019). Muscle activity of the Lumbo-pelvic-hip complex in three isometric exercises using TRX $®$ rip trainer ${ }^{\mathrm{TM}}$. Retos, 35, 216-220. Retrieved from https://recyt.fecyt.es/index.php/ retos/article/view/63922/40928

Gianakos, A., Yasui, Y., Murawski, C. D., \& Kennedy, J. G. (2016). Effects of gastrocnemius recession on ankle motion, strength, and functional outcomes: a systematic review and national healthcare database analysis. Knee Surgery, Sports Traumatology, Arthroscopy, 24(4), 1355-1364. https://doi.org/10.1007/s00167-015-3939-3

Guadagnin, E. C., da Rocha, E. S., Duysens, J., \& Carpes, F. P. (2016). Does physical exercise improve obstacle negotiation in the elderly? A systematic review. Archives of Gerontology and Geriatrics, 64, 138-145. https://doi.org/10.1016/j.archger.2016.02.008

Guadagnin, E. C., Rocha, E. S. da, Bobbert, M., Duysens, J., \& Carpes, F. P. (2020). Do older women with a fall history cross obstacles differently than older women with no fall history? Human Movement, 21(3), 47-53. https://doi.org/10.5114/hm.2020.91345

Hatfield, J., \& Murphy, S. (2007). The effects of mobile phone use on pedestrian crossing behaviour at signalised and unsignalised intersections. Accident Analysis \& Prevention, 39(1), 197-205. https:/ /doi.org/10.1016/j.aap.2006.07.001

Hermens, H. J., Freriks, B., Merletti, R., Stegeman, D., Blok, J., Rau, G., ... Hägg, G. (1999). European Recommendations for Surface ElectroMyoGraphy Results of the SENIAM project. Roessingh Research and Development.

Hsu, W.-C., Liu, M.-W., \& Lu, T.-W. (2016). Biomechanical risk factors for tripping during obstacle - Crossing with the trailing limb in patients with type II diabetes mellitus. Gait \& Posture, 45, 103-109. https:/ /doi.org/10.1016/j.gaitpost.2016.01.010

Lamberg, E. M., \& Muratori, L. M. (2012). Cell phones change the way we walk. Gait \& Posture, 35(4), 688-690. https://doi.org/10.1016/ j.gaitpost.2011.12.005

Licence, S., Smith, R., McGuigan, M. P., \& Earnest, C. P. (2015). Gait Pattern Alterations during Walking, Texting and Walking and Texting during Cognitively Distractive Tasks while Negotiating Common Pedestrian Obstacles. PLOS ONE, 10(7), e0133281. https://doi.org/ 10.1371/journal.pone.0133281

MacLellan, M. J. (2017). Modular organization of muscle activity patterns in the leading and trailing limbs during obstacle clearance in healthy adults. Experimental Brain Research, 235(7), 2011-2026. https:// doi.org/10.1007/s00221-017-4946-z

Nasar, J. L., \& Troyer, D. (2013). Pedestrian injuries due to mobile phone use in public places. Accident Analysis \& Prevention, 57, 91-95. https://doi.org/10.1016/j.aap.2013.03.021

O’Neill, M. C., Lee, L.-F., Demes, B., Thompson, N. E., Larson, S. G., Stern, J. T., \& Umberger, B. R. (2015). Three-dimensional kinematics of the pelvis and hind limbs in chimpanzee (Pan troglodytes) and human bipedal walking. Journal of Human Evolution, 86, 32-42. https://doi.org/10.1016/j.jhevol.2015.05.012

Pan, H.-F., Hsu, H.-C., Chang, W.-N., Renn, J.-H., \& Wu, H.-W. (2016). Strategies for obstacle crossing in older adults with high and low risk of falling. Journal of Physical Therapy Science, 28(5), 1614-1620. https://doi.org/10.1589/jpts.28.1614

Pijnappels, M., Bobbert, M. F., \& Van Dieën, J. H. (2006). EMG modulation in anticipation of a possible trip during walking in young and older adults. Journal of Electromyography and Kinesiology, 16(2), 137143. https://doi.org/10.1016/j.jelekin.2005.06.011

Shumway-Cook, A., Woollacott, M., Kerns, K. A., \& Baldwin, M. (1997). The effects of two types of cognitive tasks on postural stability in older adults with and without a history of falls. Journals of Gerontology - Series A Biological Sciences and Medical Sciences, 52(4). https:// doi.org/10.1093/gerona/52A.4.M232

Valencia, O., Araneda, O., Cárcamo, M., Carpes, F., \& Guzmán-Venegas, R. (2018). Relationship between lower limb anthropometry and temporo-spatial parameters in gait of young adults. Retos, 33, 1258. Retrieved from https://recyt.fecyt.es/index.php/retos/article/ view/58136/36652

Valencia, O., Cristi, I., Ahumada, D., Meza, K., Salas, R., Weinstein, A., \& Guzmán-Venegas, R. (2020). The initial impact with forefoot increases the muscular activity of gastrocnemius during running. A quantitative study of electromyographic activity. Retos, 38, 271-275. Retrieved from https://recyt.fecyt.es/index.php/retos/article/view/73955/49644

Wells, H. L., McClure, L. A., Porter, B. E., \& Schwebel, D. C. (2018). Distracted Pedestrian Behavior on two Urban College Campuses. Journal of Community Health, 43(1), 96-102. https://doi.org/ 10.1007/s10900-017-0392-X

Yogev-Seligmann, G., Rotem-Galili, Y., Mirelman, A., Dickstein, R., Giladi, N., \& Hausdorff, J. M. (2010). How Does Explicit Prioritization Alter Walking During Dual-Task Performance? Effects of Age and Sex on Gait Speed and Variability. Physical Therapy, 90(2), 177-186. https://doi.org/10.2522/ptj.20090043 